\title{
A Model of a Permanent Magnet Axial-Flux Machine Based on Lie's Symmetries
}

\author{
Luiz Tiaraju R. Loureiro ${ }^{1}$, Aly F. Flores Filho ${ }^{1}$, Jorge R. S. Zabadal ${ }^{2}$, and Roberto P. Homrich ${ }^{1}$ \\ ${ }^{1}$ Department of Electrical Engineering, Federal University of Rio Grande do Sul, Porto Alegre 90035-190, RS Brazil \\ ${ }^{2}$ Department of Nuclear Engineering, Federal University of Rio Grande do Sul, Porto Alegre 90035-190, RS Brazil
}

A permanent magnet axial-flux machine was designed, built, and tested. Numerical and analytical models were developed for the machine and comparisons were carried out. The analytical models rely on Lie's symmetries and the comparison results will be presented.

Index Terms-Axial-flux machines, Lie's symmetries, permanent magnets, Torus machine.

\section{INTRODUCTION}

$\mathbf{T}$ HE axial-flux machines tend to present a larger air gap than the radial ones, therefore, requiring higher excitation. That can be provided by permanent magnets. Several papers have studied such machines in constructive, control, and in modeling aspects [1], [3]-[6]. For further studies concerning a new analytical model, an axial-flux machine with rated rotation of 450 $\mathrm{r} / \mathrm{min}$, torque of $3 \mathrm{~N} \cdot \mathrm{m}$, and an outside diameter of $0.16 \mathrm{~m}$ was built. A finite element software was employed to carry out the numerical simulation of its flux distribution. Analytical models for the windings and permanent magnets inductions were developed. Those models rely on the Lie's symmetries, which are variable changes that maintain the form of differential equation [2]. This kind of variable change transforms an exact solution of a differential equation into another solution of the original differential equation, with more arbitrary elements. The latter are elements that present flexibility to satisfy boundary conditions. The solution with the Lie's symmetries is a different way to solve a cylindrical 3-D problem, because it avoids solutions using Bessel's functions and spatial harmonics, which require a very large number of terms to converge. Along with the permanent magnet induction, the electromotive force and the torque were also computed.

\section{DESCRIPTION OF TORUS MACHINE}

The Torus machine that was studied is a disc-type axial-flux machine, with a stator mounted between two external rotors. Its construction is rather simple, compact, and has reduced axial length. That is required by applications with volume restrictions.

The machine has a toroidal stator with eighteen coils that are connected in three groups with six coils in series, which are energized sequentially. Each rotor has six permanent magnets with alternating polarity. The poles of the permanent magnets on one rotor are mirrored by the ones at the same angular position on the other rotor. The magnetic inductions of permanent magnets interact with the currents of the stator coils, and torque is produced. The excitation of the coils' groups is carried out through a converter that energizes the coils sequentially. By that way,

Digital Object Identifier 10.1109/TMAG.2008.2002196
TABLE I

TORUS MACHINE CHARACTERISTICS

\begin{tabular}{lclc}
\hline \hline Quantity & Value & Quantity & Value \\
\hline Rotation & $450 \mathrm{RPM}$ & Number of poles & 6 \\
Torque & $3 \mathrm{Nm}$ & Number of phases & 3 \\
Outside diameter & $0.16 \mathrm{~m}$ & Number of coils & 18 \\
Axial length & $0.0545 \mathrm{~m}$ & Number of coils per phase & 6 \\
\hline \hline
\end{tabular}

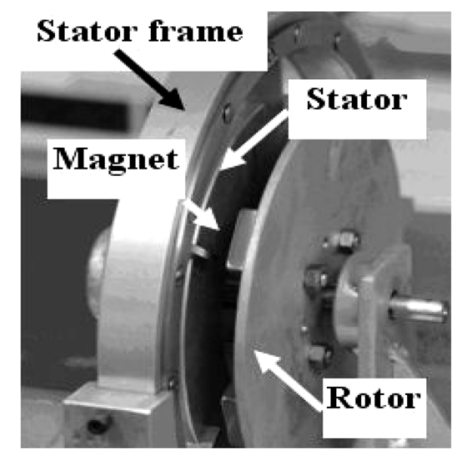

Fig. 1. Cores, magnets, and frame.

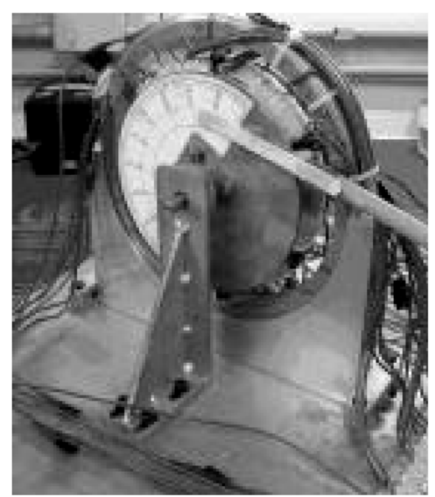

Fig. 2. Assembled machine.

the stator magnetic induction moves along with the permanent magnet magnetic induction.

The main characteristics of Torus machine are presented in Table I.

Fig. 1 shows the frame, cores and permanent magnets, while Fig. 2 shows the complete machine.

As far as a machine section is considered, the flux lines point from a north pole and across the air gap, which includes the windings. Subsequently, they enter the stator core and reach as 


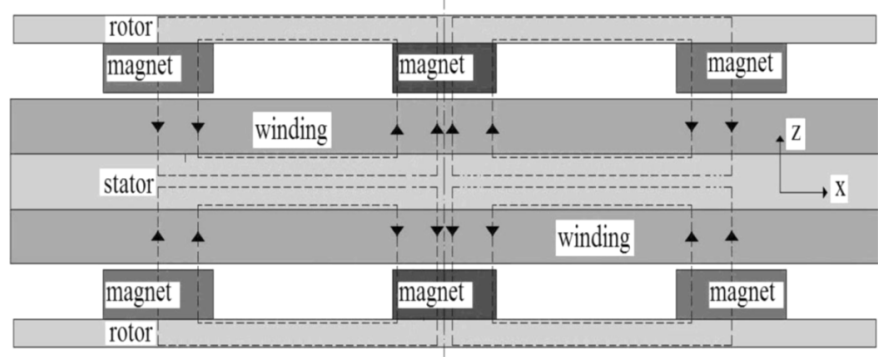

Fig. 3. Flux lines in machine's section.

far as the next pole alignment, leave the stator and cross the air gap again. After leaving the air gap, the flux lines point towards the south pole of an adjacent permanent magnet, leave it through the north pole and enter the rotor core. In the rotor, the flux lines describe a pole pitch. Fig. 3 shows a machine section with the indication of the trajectory of the flux lines.

Every driving cycle, the converter energizes two phases, while the third one remains de-energized. In the following cycle, considering the direction of rotation, the first phase is de-energized and the third one is energized.

The converter comprehends three parts: the control circuit, with a microprocessor that executes the driving cycles; the interface that isolates the control circuit from the power circuit; and the power circuit, which has power transistors to energize the windings.

\section{ANALYTICAL MODEL AND VALUES COMPARISON}

\section{A. Windings' Magnetic Induction}

The model for the windings' magnetic induction was developed in cylindrical coordinates $r, \theta$, and $z$, because the cylindrical structure of the Torus machine. As there are currents in windings, the magnetic behavior of windings can be described by

$$
-\nabla(\nabla \circ \vec{A})+\nabla^{2} \vec{A}=-\mu \vec{J}
$$

where $A$ is the magnetic potential vector, $\mu$ is the magnetic permeability, and $J$ is the current density vector.

When one applies the Coulomb's gage, the first term of the left-hand side of (1) can be set to zero, as seen in (2)

$$
\nabla^{2} \vec{A}=-\mu \vec{J}
$$

The development of (2) results in three equations

$$
\begin{aligned}
-\frac{\frac{\partial^{2} A_{r}}{\partial \theta^{2}}+r^{2} \frac{\partial^{2} A_{r}}{\partial z^{2}}}{r^{2}} \vec{u}_{r} & =\mu_{o} J_{r} \vec{u}_{r} \\
\frac{-\frac{\partial A_{r}}{\partial \theta}+\left(\frac{\partial^{2} A_{r}}{\partial \theta \partial r}\right) r}{r^{2}} \vec{u}_{\theta} & =0 \\
\frac{\frac{\partial A_{r}}{\partial z}+\left(\frac{\partial^{2} A_{r}}{\partial z \partial r}\right) r}{r} \vec{u}_{z} & =0 .
\end{aligned}
$$

An expression for $A_{r}$ is obtained by solving (4) and (5) through an order reduction and comparing the solutions. The

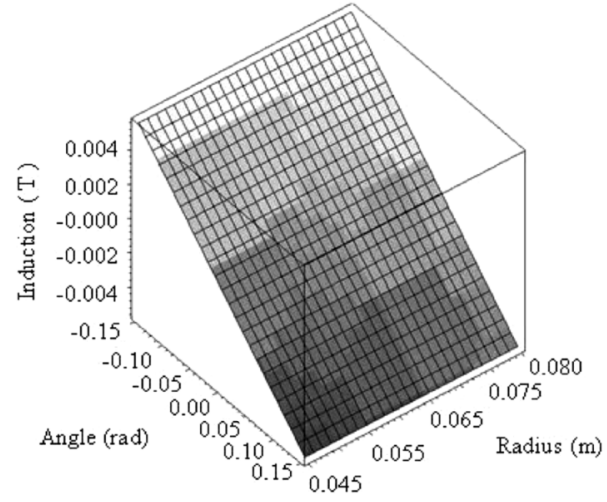

Fig. 4. $Z$-component of winding magnetic induction.

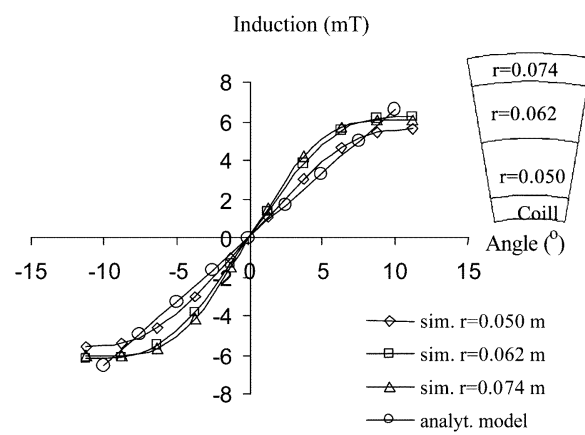

Fig. 5. Winding magnetic induction.

expression of the magnetic vector potential is derived by replacing it in (3) and solving the resulting equation

$$
\vec{A}_{r}=\frac{1}{r}\left(\Gamma \frac{z^{2}}{2}+C_{4} z+C_{5}\right) \vec{u}_{\theta}+r\left(-\Gamma \frac{\theta^{2}}{2}+C_{6} \theta+C_{7}\right) \vec{u}_{z} .
$$

With the aid of the boundary conditions, the magnetic induction of the windings is computed

$$
\vec{B}_{e f}=\frac{1}{r}\left(\Gamma z+C_{4}\right) \vec{u}_{\theta}+\left(\Gamma \theta-C_{6}\right) \vec{u}_{z} .
$$

Equation (8) is obtained by applying a Lie's translation symmetry [2] admitted by (7)

$$
\vec{B}_{e f}=\frac{1}{r}\left(\Gamma z+C_{4}\right) \vec{u}_{\theta}+\left(\Gamma \theta-C_{6}\right) \vec{u}_{z}+C_{8}
$$

where $B_{e f}$ is the air gap induction produced by the windings, $\Gamma, C_{4}, C_{5}, C_{6}, C_{7}$, and $C_{8}$ are constants.

The $z$-component of winding magnetic induction is shown in Fig. 4.

The calculated and simulation figures of the $z$-component of winding magnetic induction along the arcs with constant radii equals to $0.050,0.062$, and $0.074 \mathrm{~m}$ are shown in Fig. 5 .

\section{B. Permanent Magnets' Magnetic Induction}

The model of the permanent magnets' magnetic induction was developed in rectangular coordinates $x, y$, and $z$, based on

$$
\vec{B}=\mu_{o} \vec{H}=\mu_{o} \nabla \Psi
$$


where $B$ is the magnetic induction vector produced by the permanent magnets, $H$ is the magnetic field intensity, and $\Psi$ is the scalar magnetic potential.

The air gap scalar magnetic potential was modeled by means of Gaussian curves, according to

$$
\begin{aligned}
& f_{1}=\left(e^{-x^{2} / 4 k_{1} t}\right) / \sqrt{4 \pi k_{1} t} \\
& f_{2}=\left(e^{-y^{2} / 4 k_{1} t}\right) / \sqrt{4 \pi k_{1} t}
\end{aligned}
$$

where $k_{1}$ is a curve parameter.

The Op operator is the 2-D Laplacian minus the time derivative

$$
\mathrm{Op}=k_{1}\left(\frac{\partial^{2} f}{\partial x^{2}}+\frac{\partial^{2} f}{\partial y^{2}}\right)-\frac{\partial f}{\partial t} .
$$

The definition of $f=f_{1}+f_{2}$ and the application of Op in the function $f$ results in an identity. As the magnetic transient is very short, (11) represents the Laplacian of the sum of $f_{1}$ and $f_{2}$.

When a variable change in $f_{1}$ is introduced

$$
f a_{1}=\exp (-a / \tau) /(2 \sqrt{\tau})
$$

with $a$ as an auxiliary variable, while $t$ is a structure parameter.

The auxiliary variable $a$ can be replaced by $x$ and $y$ with their respective displacements $x_{o}, y_{o}$, and $y_{1}$. The displacements are associated with the functions' maximum and the dominium center in the coordinate system. With the changes of variables, four curves are generated to form

$$
\begin{aligned}
f a_{2}= & \frac{a_{2}}{\sqrt{\tau}} \exp \left(\frac{a_{1}}{\tau}\right)\left(\exp \left(x+x_{o}\right)^{2}+\exp \left(y+y_{o}-y_{1}\right)^{2}\right. \\
& \left.+\exp \left(x-x_{o}\right)^{2}+\exp \left(y-y_{o}-y_{1}\right)^{2}\right)
\end{aligned}
$$

where $a_{1}$ and $a_{2}$ are constants.

By replacing $x=x+j z$ and $y=y+\mathrm{j} z$ in (13), it makes the combined function become a function of $x, y$, and $z$. The resulting equation is still a solution of Laplace's equation. The scalar magnetic potential is obtained by replacing the numeric value of $\tau$ and extracting the real part of the resulting equation. The expression of the permanent magnet induction is derived by replacing the scalar magnetic potential in (9) and applying a scale Lie's symmetry.

Fig. 6 shows the magnetic induction at $0.5 \mathrm{~mm}$ from the permanent magnet surface in the air gap.

The values of the permanent magnet induction corresponding to the simulation, the analytical model and the measurements are shown in Fig. 7.

\section{Electromotive Force}

The electromotive force $e$ was calculated by the Faraday's law

$$
e=-\int_{S} \frac{\partial \vec{B}}{\partial t} d \vec{S}
$$

where $S$ is the section covered by the displacement of conductors' projection on a permanent magnet polar face.

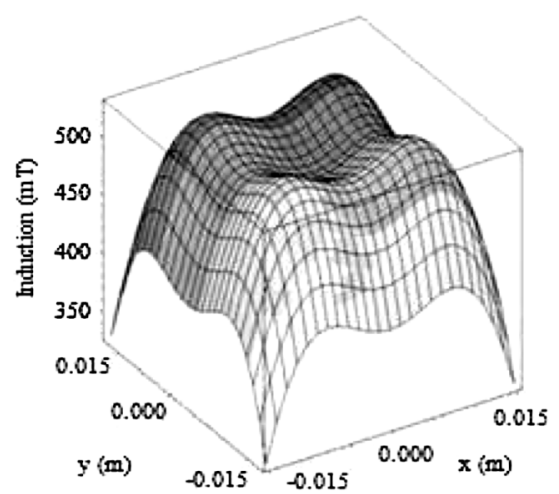

Fig. 6. Magnetic induction near permanent magnet's surface.

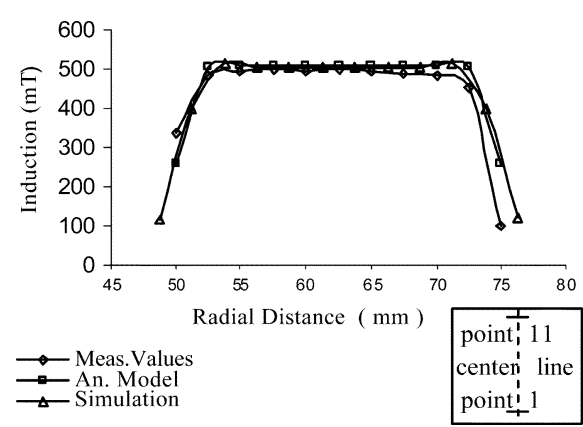

Fig. 7. Magnets magnetic induction.

As the magnetic induction is a smooth and continuously derivable function, (14) can be written as

$$
e=-\frac{d}{d t}\left(\int_{S} \vec{B} \circ d \vec{S}\right) .
$$

Since the magnetic induction is perpendicular to the plan of conductors, the gradient of the scalar magnetic potential is equal to the $z$-derivative of that quantity

$$
e=-\frac{d}{d t}\left(\int_{S} \frac{d \Psi}{d z} d S\right)
$$

The operators order can be inverted in (16) and $d S$ can be developed, so the expression of electromotive force can be written as

$$
e=-\frac{d}{d t} \frac{d}{d z} \iint \Psi d x d y
$$

The integrals of the scalar magnetic potential with respect to $x$ and to $y$ result in

$$
\begin{aligned}
f a_{3}= & \frac{a_{2} \sqrt{\pi}}{2}\left(\operatorname{erf}\left(\frac{a_{1}\left(x+x_{o}\right)}{\sqrt{-a_{1} \tau}}\right) y\right. \\
& +\operatorname{erf}\left(\frac{a_{1}\left(y-y_{1}+y_{o}\right)}{\sqrt{-a_{1} \tau}}\right) x+\operatorname{erf}\left(\frac{a_{1}\left(x-x_{o}\right)}{\sqrt{-a_{1} \tau}}\right) y \\
& \left.+\operatorname{erf}\left(\frac{a_{1}\left(y-y_{1}-y_{o}\right)}{\sqrt{-a_{1} \tau}}\right) x\right) / \sqrt{-a_{1}} .
\end{aligned}
$$




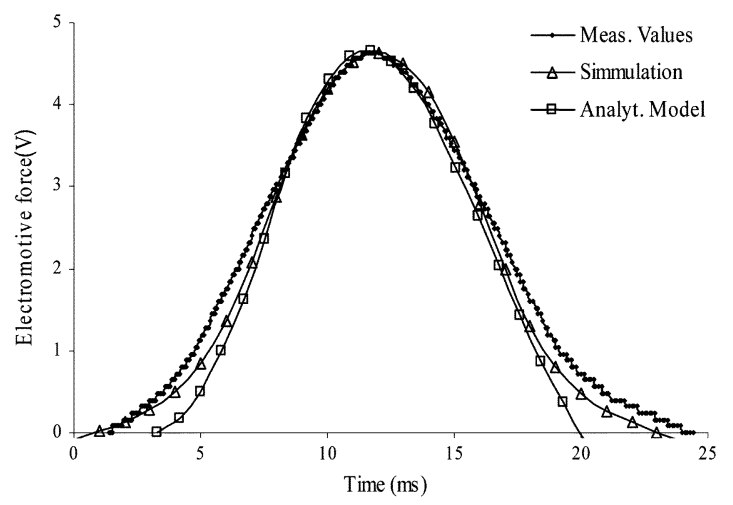

Fig. 8. Electromotive force.

In order to obtain the electromotive force, two variable changes were carried out: $x=x+j z$ and $y=y+j z$. The real part was extracted and other two variable changes were made: $x=r \cos \omega t$ and $y=r \sin \omega t$. The time derivative of the last expression results in the expression of the electromotive force.

Fig. 8 shows the analytical, simulation, and measurements results of the electromotive force.

\section{Torque}

The force produced by the interaction between the magnetic induction of the permanent magnets and the current in the coils located on the permanent magnet projection can be calculated by Lorentz's force law, i.e.,

$$
d \vec{F}=\vec{J} \times \vec{B} d V
$$

where $F$ is the force produced by the interaction of the current density and the permanent magnets' magnetic induction.

Based on the geometric parameters of the coils, the current density in the Torus machine can be expressed as

$$
J=\frac{N I}{\theta_{b} h_{b}} \frac{1}{r}=\frac{k}{r}
$$

where $N$ is the number of turns in each coil, $I$ is the current in each coil [A], $\theta_{b}$ is the angle that describes the coil arc, $h_{b}$ is the coil's thickness, and $r$ is the $r$-coordinate of the reference system.

The differential $d F$ becomes (21) when one replaces the current density in (19)

$$
d \vec{F}=\frac{k}{r} \vec{r} \times \vec{B} d V
$$

The torque differential is equal to the force differential multiplied by $r$

$$
d T=r \frac{k}{r} B d V
$$

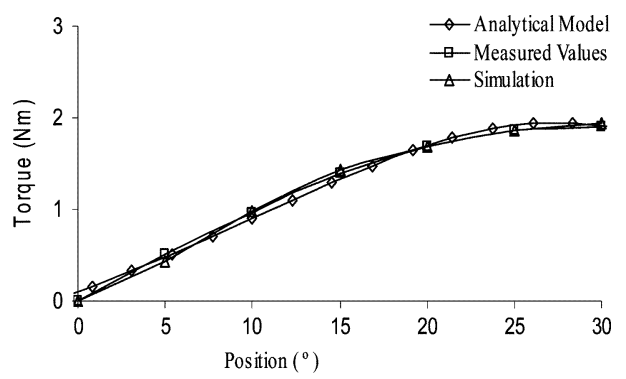

Fig. 9. Torque of the Torus machine.

The torque is obtained by the integration of (22)

$$
T=k \iiint B d x d y d z
$$

The torque expression becomes (24) when the definition of the scalar magnetic potential is introduced in (23)

$$
T=k \iiint d \Psi d x d y \text {. }
$$

Once the integrations were carried out, the $z$-coordinate is introduced through the variable changes $x=x+j z$ and $y=$ $y+j z$, the real term is extracted, and the result obtained is the torque expression.

Fig. 9 presents the torque values from the analytical model, the measured and the simulated ones.

\section{CONCLUSION}

The studies carried out with the Torus machine produced analytical and numerical results that are compatible with the values of the measurements. By that it is demonstrated the Lie's symmetries can be applied in electromagnetic systems, since they are a very versatile method for analyzing complex differential equations.

\section{REFERENCES}

[1] E. Spooner and B. J. Chalmers, "Torus: A slotless, toroidal-stator, permanent-magnet generator," Proc. Inst. Elect. Eng.-Electric Power Appl., vol. 139, no. 6, pp. 497-506, Nov. 1992.

[2] P. J. Olver, Applications of Lie Groups to Differential Equations., 2nd ed. New York: Springer-Verlag, 2000.

[3] J. F. Gieras, R. J. Wang, and M. J. Kamper, Axial Flux Permanent Magnet Brushless Machines.. Dordrecht: Kluwer Academic , 2004.

[4] Y. N. Zhilichev, "Three-dimensional analytic model of permanent magnet axial flux machine," IEEE Trans. Magn., vol. 34, no. 6, pp. 3897-3901, Nov. 1998.

[5] M. Aidin, S. Huang, and T. A. Lipo, "Design and 3D electromagnetic field analysis of non-slotted and slotted TORUS type axial flux surface mounted permanent magnet disc machines," in Conf. Rec. Electric Mach. Drives Conf., Cambridge, NY, 2001, pp. 645-651.

[6] A. Parviainen, M. Niemelä, and J. Pyrhönen, "Modeling of axial flux permanent-magnet machines," IEEE Trans. Ind. Appl., vol. 40, no. 5, pp. 1333-1340, Sep.-Oct. 2004, New York.

Manuscript received March 02, 2008. Current version published December 17, 2008. Corresponding author: L. T. R. Loureiro (e-mail: tiaraju@ufrgs.br). 